\title{
MISMATCH NEGATIVITY RELATED TO UPDATING INFORMATION IN OBJECT FILES
}

\author{
Masayoshi NAGAI ${ }^{1)}$ and Akihiro YAGI ${ }^{2)}$ \\ ${ }^{1)}$ National Institute of Advanced Industrial Science and Technology, Japan, and ${ }^{2)}$ Kwansei Gakuin University, Japan
}

\begin{abstract}
The present study investigated the process of updating or replacing the contents in object files using behavioral (reaction time) and psychophysiological (mismatch negativity) measures. Two moving letters were presented, and participants responded the target letter's identity as quickily as possible. In the coherent condition, the two letters moved downward in natural way, keeping their spatiotemporal coherence. In the incoherent conditions, the two letters were replaced by each other or by new and different letters. In these cases, the spatiotemporal coherence was violated and participants had to update the information about each moving letter. The results showed that the reaction times were longer in the incoherent conditions than in the coherent condition. Mismatch negativity, which reflected the presentation of unpredictable events, was observed in the incoherent conditions. We propose that mismatch negativity is one measure to assess the efficiency of interface devices in daily lives.
\end{abstract}

Key words: object file, mismatch negativity, expectation

In their daily lives, people encounter numerous kinds of visual displays and interfaces for getting useful information and manipulating devices. One important example is a car navigation system. Drivers are required to perform many tasks and process various kinds of information in using it: inputting a goal location, perceiving visual letters or landmarks on the visual display, keeping information in working memory, updating that information when it is changed, and so on. It would be easy to use a car navigation system if the driver could focus only on manipulating the navigation device all the time during driving. However, drivers have to allocate their primary processing resources to their driving, to observing the constantly changing road conditions and operate the vehicle. As a sub-primary task, using a navigation system can be difficult because the opportunities to see the display and the processing resources are limited. Drivers must use some visual memory for representing the current position of the vehicle related to the display map, and for predicting the updated vehicle position on the map since their last glance at the display.

The present study addresses these representational and updating processes. One important concept for understanding these processes is the "object file" (Kahneman \&

This study was supported by the Research Fellowship of Japan Society for the Promotion of Science for Young Scientists (200201154).

Correspondence concerning this article should be addressed to Masayoshi Nagai, Institute for Human Science and Biomedical Engineering, National Institute of Advanced Industrial Science and Technology, AIST Tsukuba Central 6, 1-1-1 Higashi, Tsukuba, Ibaraki 305-8566, Japan (e-mail: masayoshi-nagai (a)aist.go.jp).

${ }^{2)}$ Department of Psychology, Kwansei Gakuin University, Uegahara, Nishinomiya, Hyogo, 662-8501, Japan. 


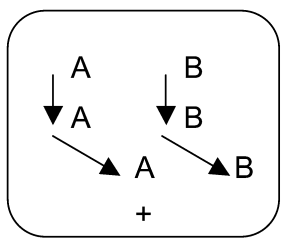

A) Slide

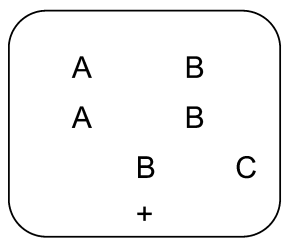

B) Cross

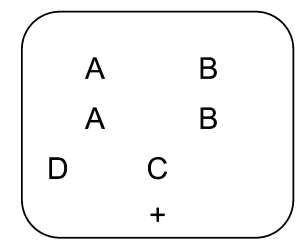

C) New

Fig. 1. Stimulus conditions in this study. Stimulus consisted of a three-frame sequence of two letters on each trial. In Figure 1A, the arrows show an example of the motion path of the two letters. Note that in the actual stimulus presentation these arrows were not shown.

Treisman, 1984). The object file is a temporary episodic representation of objects, which is distinct from the representations stored in long-term memory (Kahneman, Treisman, \& Gibbs, 1992). Each object file contains information about particular objects in a visual scene, and is addressed by its location at a particular time, not by any feature or identifying label. An object file collects sensory information, updates it as the sensory situation changes, and may be discarded when the object disappears from view. The object-specific priming effect is considered to be evidence for the object file.

In the present experiment, two letters move in three temporal frames, as shown in Fig. 1. In the slide condition, the two letters move while maintaining their spatiotemporal continuity (Fig. 1A). In other words, the letter A is always at the left side and the letter B is always at the right side. Therefore, each object file contains consistent information for the complete temporal sequence. However, in the other two conditions, the spatiotemporal continuity is disrupted (Fig. 1B and C). In the cross condition, the relative positions of the two letters is changed from the second to the third frame. In this case the relative spatial information in the object file has to be changed even though the objects are the same. In the new condition, new two letters appear in the third frame. So, the object file information has to be replaced by the newly presented letters. Object-specific priming was operationally defined as giving a faster response for identifying the letter above the fixation cross in the case where the objects exhibited coherent spatiotemporal continuity (the slide condition) compared to the non-coherent cases (the cross and new conditions).

This study examined the process of updating information in the object file using both behavioral and psychophysical measures. Reaction time was the behavioral measure for evaluating the updating process. For the psychophysiological measure, we utilized one of the event-related brain potential (ERP) components: mismatch negativity (Kutas \& Hillyard, 1980a, 1980b). Mismatch negativity (also called N400) is a negative ERP component observed around a parietal site when an odd word in a context was presented. Kutas and Hillyard (1984) found that mismatch negativity was also elicited by non-odd words, and its amplitude was related to the prediction of subsequently presented words. For example, when a participant's prediction of a subsequently presented word was close to the actually presented word, the mismatch negativity was small. In contrast, when the actually presented word varied greatly from the prediction, a much larger magnitude of 
mismatch negativity was elicited. We used mismatch negativity for evaluating the participants "on-line" prediction about the content in an object file. We predicted larger mismatch negativity when the object-file information was updated with an "unpredictable content".

\section{METHOD}

\section{Participants:}

Nineteen undergraduate students at Kwansei Gakuin University volunteered to participate in the experiment. All the participants had normal or corrected-to-normal vision. They were naive as to the present hypothesis.

\section{Apparatus:}

Stimuli were presented on a 14-inch CRT monitor (NEC, PC-KD852) controlled by a computer (NEC, PC-9801VX). The monitor was placed $57.3 \mathrm{~cm}$ from the participant's eyes. Responses were inputted to the computer with a switch box (Japan Assembler, PC98SWB2), which had four response keys labeled as A, B, $\mathrm{C}$, and D.

\section{Stimuli and Task:}

Four white capital letters, A, B, C, and D $\left(26.0 \mathrm{~cd} / \mathrm{m}^{2}\right)$, against a black background $\left(2.0 \mathrm{~cd} / \mathrm{m}^{2}\right)$ were used as stimuli. The size of these letters was within 0.29 degree wide and 0.23 degree high in visual angle. On each trial, participants saw a three- frame sequence of two letters (Fig. 1). In the first frame, two letters were randomly selected and presented for $150 \mathrm{~ms}$; the two letters were horizontally separated by 0.34 degree from each other. After a $40 \mathrm{~ms}$ blank screen, the same letters were presented for $150 \mathrm{~ms}$ at 0.40 degree below their first frame locations. After another $40 \mathrm{~ms}$ blank screen, the target letters were presented for $150 \mathrm{~ms}$. From the second to the third frame, the letters moved to the lower right or to the lower left (by 0.40 degree downward and 0.63 degree rightward or leftward). One of these two directions was randomly chosen for each trial. The target was one of the letters presented 0.40 degree above the fixation point $(0.29$ degree wide and 0.17 degree high). The presented letters in the third frame were selected depending on the condition. In the slide condition, the horizontal spatial relationship of the two letters was unchanged from the second to the third frame. In other words, using the example of Fig. 1, the letter A was always presented at the left side and the letter B was presented at the right side throughout the first to the third frame. In the cross condition, the spatial relationship was changed. For example, in the third frame, the letter A was now presented at the right side instead of the left side, and the letter B was presented at the left side. In the new condition, two letters, which were different from the first two frames, were presented; in this example the letters $\mathrm{C}$ and $\mathrm{D}$ would be presented.

Participants were asked to respond, as quickly as possible using the switch box, which letter was presented above the fixation point at the third frame. At the beginning of each trial a green asterisk "*" (20.0 $\left.\mathrm{cd} / \mathrm{m}^{2}\right)$ changed to the white fixation mark "+” $\left(26.0 \mathrm{~cd} / \mathrm{m}^{2}\right)$ and was presented for $500 \mathrm{~ms}$. Then the sequence of the two letters started. After the sequence presentation, the plus fixation point was changed into the green asterisk. The inter-trial interval was $2.0 \mathrm{~s}$. After two practice blocks of 16 trials, participants performed 12 experimental blocks of 16 trials. The three conditions were intermixed in each block. Participants were instructed to avoid moving their eyes, body, and eye blinks during each trial to reduce artifacts in the recording devices.

\section{EEG Recording:}

The EEGs were recorded from two sites: Pz (according to the 10-20 system; Jasper, 1958), and Oz (according to the American Electroencephalographic Society, 1991). Linked earlobes were used as the reference in order to control the artifact from eye / eye-lid eye movements subordinate to the saccade. The ground lead was attached to the midline forehead. Eye movements were recorded by means of EOGs. A pair of $\mathrm{Ag}-\mathrm{AgCl}$ electrodes was placed above the right eye and below the left eye. The EEGs and EOGs were 
amplified with differential amplifiers (Nihon Kohden, AB-621G) at a time constant of $2.0 \mathrm{~s}$ and the high frequency cut off was set at $50 \mathrm{~Hz}$. These signals were digitized every $4 \mathrm{~ms}$ with an A-D converter (TEAC PS-9351).

\section{EEG Data Analyses:}

EEGs without artifacts between $480 \mathrm{~ms}$ before to $800 \mathrm{~ms}$ after the target presentation were averaged for each condition. Data from the trials where the responses were incorrect or the reaction times (RT) were over $900 \mathrm{~ms}$ were not analyzed. The number of trials for averaging in each condition was at least 47, and the mean was 58.9. Five of the nineteen participants showed severe artifacts like alpha wave and muscle potentials. Their data were not analyzed for computing event-related potentials.

\section{RESULTS}

$R T$

The mean RTs and standard deviations for each condition are shown in Table 1. Trials where the RT was over $900 \mathrm{~ms}$. were eliminated as outliers. The RTs were analyzed in an ANOVA, and a significant main effect for condition was found $(F(2$, $26)=204.05)$. Post hoc Tukey's HSD tests $(p<.05)$ showed that the mean RTs for all of the 3 conditions significantly differed. The mean RT was the shortest in the slide condition, which was significantly shorter than the new condition, which was significantly shorter than the cross condition.

\section{Event Related Potential (ERP) Analysis}

The baselines were computed as the mean of all data points from 480 to $4 \mathrm{~ms}$ prior to the target presentation. Fig. 2 shows the mean ERP data for sites Pz and Oz. There is a clear difference in the latency at 300-400 ms among the conditions, which reflects mismatch negativity. The mean amplitudes in each condition for each site are summarized in Table 2. N400-like decreases in potential were observed in the cross and new conditions compared to the slide condition; this decrease was more severe in the cross condition than in the new condition. The averaged potentials between 300-400 ms for each recorded site were analyzed in an ANOVA and a significant main effect of condition was found (Pz: $F(2,26)=1423, p<.001$; Oz: $F(2,26)=16.12, p<.001)$. Post hoc Tukey's HSD tests $(p<.05)$ showed that for the $\mathrm{Pz}$ and $\mathrm{Oz}$ sites, the averaged potentials in the cross and new conditions were smaller (i.e., more negatively shifted) than in the slide condition. For the $\mathrm{Oz}$ site, the averaged potential in the cross condition was smaller than in the new condition.

Table 1. Mean and Standard Deviation of Reaction Time in Each Condition (ms)

\begin{tabular}{|c|c|c|c|c|c|}
\hline \multicolumn{2}{|c|}{ slide } & \multicolumn{2}{|c|}{ cross } & \multicolumn{2}{|c|}{ new } \\
\hline$M$ & $S D$ & $M$ & $S D$ & $M$ & $S D$ \\
\hline 510.71 & 41.21 & 578.79 & 41.89 & 567.42 & 40.13 \\
\hline
\end{tabular}



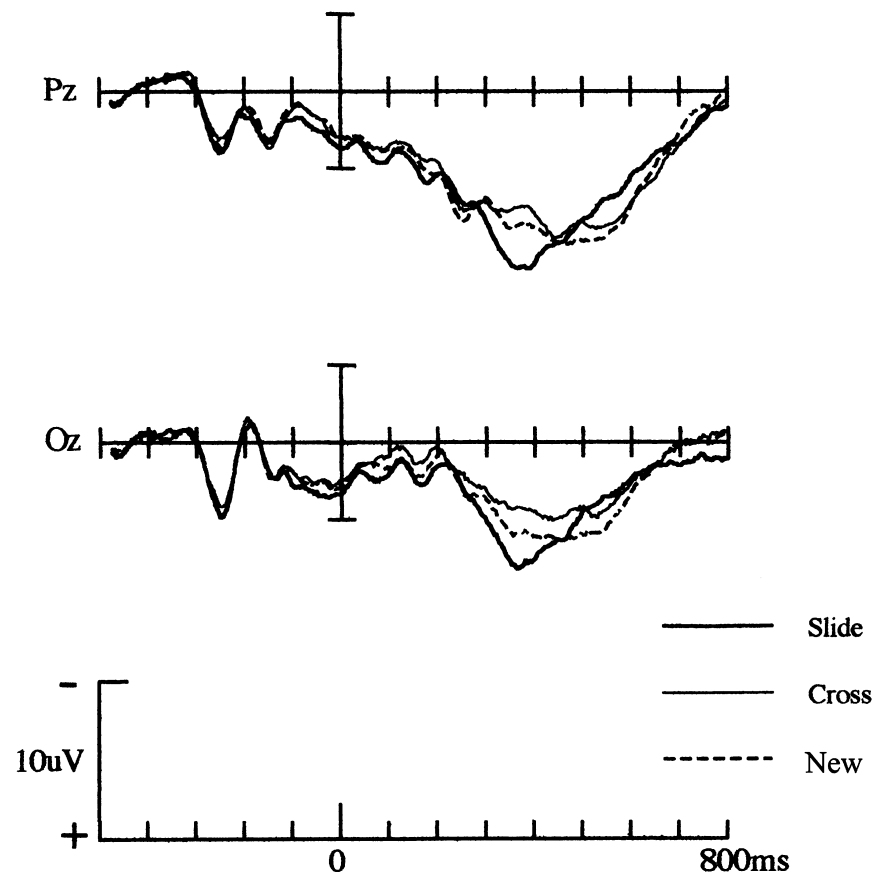

Fig. 2. Event related potentials (ERPs) in $\mathrm{Pz}$ and $\mathrm{Oz}$ in each condition. Horizontal scale shows the time course relative to the onset of the target presentation. Vertical scale shows 10 micro volt (i.e., plus and minus 5 micro volt).

Table 2. Averaged Amplitude in ERP Between 300-400 ms in Latency (micro volt)

\begin{tabular}{cccccccc}
\hline & $\mathrm{Pz}$ & & & \multicolumn{3}{c}{$\mathrm{Oz}$} \\
\cline { 1 - 2 } \cline { 5 - 7 } \cline { 5 - 7 } slide & cross & new & & slide & cross & new \\
\hline 10.02 & 8.03 & 7.18 & & 7.67 & 3.84 & 5.36 \\
\hline
\end{tabular}

Note: The averaged amplitude in RRP between 300-400 ms was used as the measure of mismatch negativity. This amplitude is separately shown for the brain loci Pz and $\mathrm{Oz}$.

\section{DISCUSSION}

The present study investigated the updating or replacing process for content in object files using behavioral (RT) and psychophysiological (mismatch negativity) measures. The behavioral data showed the longest RTs in the cross condition, and RTs in the new condition were longer than in the slide condition but shorter than in the cross condition. This trend was also observed with the psychophysiological measures: mismatch negativity was larger in the cross condition than in the new condition, and was the smallest in the slide condition. Mismatch negativity is elicited in cases where participants' future predictions or guesses fail (Kutas \& Hillyard, 1984). Therefore, the present results 
suggest that in the cross and new conditions, the participants' predictions of the upcoming spatiotemporal array of the letters did not materialize as expected. In the natural world, moving objects cannot be replaced by each other in the spatiotemporal sequence (as in the cross condition) and they cannot be replaced by new objects (as in the new condition). The difference in mismatch negativity between the cross and new conditions suggests that participants felt that the objects' spatiotemporal continuity in the cross condition was more unnatural than in the new condition. This is possible because in the cross condition participants sometimes saw two objects moving like a "crossing". For example, in Figure 1 the letter A moved from the far left to the far right and the letter B moved from the far right to the far left. However, in the other conditions, the letters did not cause this crossing motion impression. Only in the cross condition did participants see an "impossible crossing motion", and this perception might have caused a larger mismatch negativity.

The present findings suggest that mismatch negativity could be a useful measure for evaluating whether machine interfaces are efficient (i.e., safe and functional). Using mismatch negativity, we can evaluate whether visual presentations correspond to users' expectations, because unexpected changes in the display might interfere with safety if they increase reaction times. When large mismatch negativity is elicited, it could be judged that the visual presentation violates natural ways of objects' spatiotemporal continuity. In this situation, processing resources may be more extensively allocated to the strange event and, for a while, the brain is busy updating the contents of object files. Therefore, the visual system cannot efficiently attend to or process other information at that time. In designing better and safer human interfaces, we should avoid unnatural visual presentations that might cause people to ignore important information for the safe use of the interfaces. Especially in the case of driving, drivers have to pay attention to the surrounding views and should detect danger signals that potentially lead to accidents. Mismatch negativity can evaluate whether the visual presentation on an interface requires a high utilization of processing resources. In other words, it is useful to design visual information devices to avoid accidents and that can be easily used. For example, mismatch negativity can be utilized for evaluating safer car navigation systems and visual terminal devices that can be easily used by older people, children, and cognitivelyhandicapped persons.

\section{REFERENCES}

Kahneman, D. \& Treisman, A. 1984. Changing views of attention and automaticity. In R. Parasauaraman \& D. A. Davis (Eds.), Varieties of attention (pp. 29-62). New York: Academic Press.

Kahneman, D., Treisman, A., \& Gibbs, B. J. 1992. The reviewing of object files: Object-specific integration of information. Cognitive Psychology, 24, 175-219.

Kutas, M., \& Hillyard, S. A. 1980a. Event-related potentials to semantically inappropriate and surprisingly large words. Biological Psychology, 11, 99-116.

Kutas, M., \& Hillyard, S. A. 1980b. Reading senseless sentences: Brain potentials reflect semantic incongruity. Science, 207, 203-205.

Kutas, M., \& Hillyard, S. A. 1984. Brain potentials during reading reflect word expectancy and semantic association. Nature, 307, 161-163. 